\title{
Oxygen-ozone therapy in meningoencephalitis and chronic fatigue syndrome. Treatment in the field of competitive sports: case report
}

\author{
Luca Morelli, ${ }^{1-3}$ Simona Carla Bramani, ${ }^{1}$ Federico Carlo Morelli ${ }^{1}$ \\ 1 "San Fedele" Physiotherapy Polyspecialistic Centre, Longone al Segrino (CO), Italy; ${ }^{2}$ UCM United Campus of Malta, \\ Smart City, Lugano, Switzerland; ${ }^{3}$ Italian Society for Oxygen-Ozone Therapy (SIOOT), Gorle (BG), Italy
}

\begin{abstract}
Our study was born from the observation of a clinical case of a boy who arrived in the Emergency Room of our hospital for persistent hyperpyrexia, headache and prolonged emetic episodes and complaining of objective photophobia and dizziness.

The patient underwent haematochemical tests, chemical-physical examination of Chronic Fatigue Syndrome (CFS), negative for bacteria, negative for Neisseria Meningitidis, Escherichia Coli 121, Haemophilus Influenzae, Stafilococcus Pneumoniae, Stafilococcus Agalactiae and with slightly positive reaction to Pandy's test; he was subjected to neurological examination, to Nuclear Magnetic Resonance of the brain and encephalic trunk with contrast agent which resulted negative, and to EEG that showed a slightly slowed-down brain electrical activity, in right occipital region, and frontal irritative abnormalities. Given these clinical and instrumental investigations, an acute meningoencephalitis was diagnosed.

During his hospitalization, the patient was treated with intravenous antibiotic therapy and intravenous antiviral therapy for 12 days. At discharge, in the absence of specific therapy, and considering the protraction of the cephalic, dizzying, asthenic and myal-
\end{abstract}

Correspondence: Luca Morelli, Italian Society for Oxygen-Ozone Therapy (SIOOT), via Don Luigi Sturzo 2, 24020 Gorle (BG), Italy.

E-mail: info@ossigenoozono.it

Key words: Meningoencephalitis; Chronic fatigue syndrome;

Competitive sports.

Contributions: the authors contributed equally.

Conflict of interest: the authors declare no potential conflict of interest.

Funding: none.

Received for publication: 1 March 2019.

Accepted for publication: 14 March 2019.

(C) Copyright Luca Morelli et al., 2019

Licensee PAGEPress, Italy

Ozone Therapy 2019; 4:8176

doi:10.4081/ozone.2019.8176

This article is distributed under the terms of the Creative Commons Attribution Noncommercial License (by-nc 4.0) which permits any noncommercial use, distribution, and reproduction in any medium, provided the original author(s) and source are credited. gic symptoms and in relation to hematochemical and serological tests (positive for antibodies to Herpes $1 \mathrm{IgG}$ ), Epstein Barr Virus antibodies (positive for Viral Capsid Antigen IgG and IgGE BNA, for Extractable Nuclear Antigen and IgG Cytomegalovirus) he was diagnosed a Post-infectious CFS.

The patient was treated with Oxygen Ozone Rectal Insufflative Therapy on a bi-weekly basis for 4 weeks, associated with Micetrin, a dietary supplement with sweetener based on Vitamin C, Shitake, Reishi, Maitake, Cordyceps, Magnesium and SOD, continued the treatment on a weekly basis for a further 4 weeks until the complete remission of the symptoms of asthenic, neurological and clinical parameters.

\section{Introduction}

Previously, in our clinic, we treated a World Champion Athlete for Post-infectious Chronic Fatigue Syndrome (CFS) with a similar encephalitic and hematochemical clinical picture of previous toxoplasmosis infection and asymptomatic assessed mononucleosis.

Also in this observational study, after oxygen ozone systemic therapy, there was the complete remission of the asthenic-neurological symptoms and the negative results of the hematochemical and instrumental tests.

From these observations and from our clinics experience with patients with CFS, we can say that oxygen-ozone therapy is an effective therapeutic device in the neurological and functional aftermath of post-infectious meningoencephalitic syndromes. ${ }^{1-12}$

\section{Case Report}

Our study was born from a clinical case of a 14-year-old boy with persistent hyperpyrexia $\left(39^{\circ}\right.$ Celsius $)$ and refractory to paracetamol therapy, intense headache with prolonged emetic episodes, important objective dizzying symptoms, alternating with subjective dizzying symptoms that the boy described as a feeling of fall or instability associated with photophobia even in the presence of dim light.

In his medical history, the patient had been admitted one year before for similar symptoms characterized by persistent asthenia, subjective dizziness, blurred vision in postural passages, photophobia, reported drowsiness and sometimes nausea.

Hematochemical tests did not show signs of inflammation, but marked eosinophilia ( $1840 \mathrm{mmc}-20 \%$ of GB) in previous parasitosis by pinworms. At the time of admission, the search for parasites 
in the feces, including the Giardia, was negative; EEG was normal; MRI brain was normal, positive for maxillary and paranasal sinusitis. Serology was negative for Mycoplasma, Streptococcus Betahemolytic A and for Borrelia Burgdoferi. The serology for Cytomegalovirus (CMV) and Epstein Barr Virus (EBV) was positive, as for the previous infection.

During his stay he was treated for sinusopathy and discharged with diagnosis of right maxillary sinusitis and rhinosinusitis in patient with allergic diathesis and previous oxyuriasis.

In the following months, the symptomatology slowly regressed until the following year, when a similar neurological recurrence occurred.

At the beginning, the patient was admitted to hospital for clinical and instrumental investigations following hyperpyrexia refractory to therapy, and subjected to neurological examination: intact sensorium, Romberg positive in the absence of focal deficits; Nuclear Magnetic Resonance of the brain and encephalic trunk with contrast agent: no significant focal points or enhancement alterations at the brain level; normal appearance of CFS spaces and of the ventricular system; normal findings for the suprasellar region, the cerebellopontine angles and the orbits; right maxillary and frontal sinusitis pattern, bilateral ethmoidal with modest thickening of the mucosa of the left maxillary sinus.

The electroencephalography revealed a slightly slowed-down brain electrical activity in the right occipital region and mainly frontal irritative abnormalities. The hematochemical test showed normal values with white globules $6900 / \mathrm{mmc}$ and Reactive Protein C $1.65 \mathrm{mg} / \mathrm{dL}$.

A rachicentesis and a chemical-physical examination of the liqueur were performed: clear, colorless appearance, GB 110 $\mathrm{mg} / \mathrm{dL}$, protein $129 \mathrm{mg} / \mathrm{dL}$ glucose 50\% and Pandy's reaction with slight numbness. The direct bacterioscopic examination of the CFS was negative for bacteria. Bacterial antigens in CFS were found to be negative for Neisseria Meningitidis, Escherichia Coli 121, Haemophilus Influenzae, Stafilococcus Pneumoniae and Stafilococcus Agalactiae. The CFS genomic sequence analysis was negative for Herpes Simplex 1, H. Simplex 2 and Enterovirus. At hemoculture no development was assessed.

To serological tests the following results were assessed:

i) HSV Herpes Antibodies 1 IgG: positive;

ii) Herpes $2 \mathrm{IgG}$ antibodies: negative;

iii) Antibodies against Herpes 1 IgM and Herpes 2 IgM: negative;

iv) Antibodies to EBV: Viral Capsid Antigen (VCA): IgG positive; VCA IgM:_diagnostic doubts;

v) Epstein Barr Nuclear Antigen (EBNA) IgG: positive;

vi) Anti-Nucleus Antibodies (ANA): weakly positive 1/160 homogeneous (in a range between $1 / 80$ and $1 / 160$ );

vii) Anti-DNA: negative;

viii) Extractable Nuclear Antigen (ENA): positive to anti SSB and SSA (ro 52 and ro 60).

The analysis of genomic sequences on pharyngeal swab for respiratory viruses was negative. Given the results of the serological investigations, the screening was completed with the evaluation of the autoimmunity:

i) Complement: within limits;

ii) Lupus Anticoagulant (LAC): negative;

iii) Antibodies anti-Cardiolipin (ACA) IgG and IgM: negative.

The serology was also negative for Salmonella and Brucella. From these clinical and instrumental investigations, an acute meningoencephalitis was diagnosed.

During his hospitalization, the patient was treated with Ceftriaxone intravenous antibiotic therapy for 8 days and Aciclovir intravenous antiviral therapy for 12 days. At the complete remission of the febrile state, the patient was discharged with sympto- matic treatment for headache, indication to rest, hematochemical and autoimmunity controls over time, or neurological reassessment and a programmed EEG.

At the patient's discharge, in the absence of specific therapy, in consideration of the protraction of the cephalalgic, vertiginous, asthenic and myalgic symptoms and in relation to the hematochemical and serological tests positive for anti-Herpes $1 \mathrm{IgG}$ antibodies, EBV antibodies: positive for VCA IgG and EBNA IgG; ANA positive, ENA positive and CMV IgG positive, he was diagnosed a Post-infectious CFS.

The patient was treated with oxygen-ozone therapy Rectal Insufflation $150 \mathrm{cc}$ at a concentration of $35 \mathrm{mcg}$ at bi-weekly intervals for 4 weeks, continued treatment on a weekly basis for a further 4 weeks associated with Micetrin, a dietary supplement with sweetener based on Vitamin C, Shitake, Reishi, Maitake, Cordyceps, Magnesium and SOD, to double daily dosage for the first two weeks followed by single daily dosage for the entire period of treatment of the patient, until the complete remission of asthenic symptoms and regression of cephalic, vertiginous and myalgic symptoms.

\section{Follow-up}

Two months after discharge, the patient (under treatment with oxygen-ozone therapy associated with Micetrin supplement), was hospitalized for the planned clinical and neurological investigations.

i) The Neurological Objective Examination was within normal limits with a slightly positive Romberg (tendency to fall to the left) and some uncertainty in digital dexterity tests associated with nuanced bilateral tremor.

ii) The brain $3 \mathrm{~T}$ MRI was normal, with fluid material of likely inflammatory nature that occupied the right maxillary sinus, the ethmoidal cells and the frontal sinuses.

iii) The eye examination revealed no pathological findings.

iv) The otolaryngology examination did not find signs of positional peripheral vestibular suffering in progress.

v) The impedance audiometry test revealed nothing pathological. The results of the serological test of ENA were as follows:

i) Antibodies to JO_1: negative;

ii) Antibodies against RPN: negative;

iii) Antibodies to Ski 70: negative;

iv) Antibodies anti Sm: negative;

v) SSB antibodies: negative;

vi) SSA antibodies: positive with value $=8.0$ of doubtful positivity in a normal range between 7 and 10 (values lower than 7: negative; values higher than 10: positive).

The patient continued in the following months the oxygenozone therapy with rectal insufflations of $150 \mathrm{cc}$ at a concentration of $35 \mathrm{mcg}$ on a monthly basis and currently on a bimonthly basis until the next programmed Neuro-Rheumatological control, with a food supplement as maintenance.

\section{Discussion}

The symptoms attributable to the post-infectious CFS of this clinical case have similar neurological symptomatology and diagnostics (in particular the electroencephalographic picture showing frontal irritative anomalies), with our previous case study of an international athlete, who in 2010 came to our observation for reported asthenia, oppressive anxiety sensation such as air hunger, polymyalgia and general malaise such as empty head. That symptomatology lasted for about a year, after an episode of flu syn- 
drome, following a previous infection from toxoplasmosis and mononucleosis evaluated as asymptomatic.

The patient, who was followed for checks and fitness examinations for competitive sports at the C.O.N.I. Institute of Medicine and Sport Science in Rome, reported the continuation of asthenic and anxiogenic symptomatology, also at the time of the serial sport medical evaluation.

He had been subjected to neurological investigations in order to express an opinion about its sporting validity and suitability, through neurological evaluation for:

i) clinical examination: no focal signs;

ii) electroencephalographic examination with frequency and amplitude maps, showing the presence of slow anterior activity;

iii) Nuclear Magnetic Resonance brain: no focal signs;

iv) PET brain: no sign of district hypoperfusion;

v) Event-related potentials: normal for latency and extent.

Since a pituitary-adrenal hyperactivity was found in the laboratory tests, the patient was subjected to further clinical and laboratory tests that did not attribute this hyperactivity to endocrine abnormalities. The athlete had also performed a stress profile that revealed an increased level of neurovegetative activity, and a personality profile associated with high levels of anxiety. The patient was treated for the described symptoms with common analgesics as needed and with anxiolytics that he took only for one month without benefit.

Since there were no contraindications to the continuation of the specific sporting activity, the suitability for competitive sport activity was confirmed.

From the clinical, instrumental and laboratory investigations carried out at the follow up examination at our "San Fedele" Physiotherapy Polyspecialistic Centre and from the persistence of the symptoms described, a diagnosis was made that could be ascribed to the symptomatologic complex of the CFS, also in relation to the infectious etiological onset of the previous toxoplasmosis, mononucleosis and influenza syndrome.

In view of the poor effectiveness of the antalgic and anxiolytic antidepressant therapy taken by the patient, an initial bi-weekly cycle of 4 sessions of oxygen-ozone therapy was performed by auto-hemo therapy at a concentration of $35 \mathrm{mcg}$ per $100 \mathrm{cc}$ of blood, subject to the treatment authorization requested from C.O.N.I and the competent authorities National Anti Doping Organization (NADO), CEFT - Italian NOC TUEC by means of the F49 Therapeutic Use Exemptions Application (TUE) forms in force since 9 December 2009 and supported by clinical, laboratory and diagnostic certification.

At the initial remission of the asthenic, anxiogenic, psychogenic and confusing symptoms of the sensory system, the cycle was continued with rectal insufflations of oxygen-ozone at a concentration of $35 \mathrm{mcg}$ for $150 \mathrm{cc}$ mono weekly with a progressive increase in latency at fortnightly, monthly, bi and quarterly administration in relation to the patient's training periods and transfers for a total of 12 sessions, until the complete remission of the disease and normalization of the endocrine hematochemical examinations.

In 2011, our athlete won a further World Champion title, increasing the total number of his Gold Medals, once again bringing Italy to the top of the world sport.

\section{Conclusions}

The name Chronic Fatigue Syndrome was chosen by a group of experts in 1988 because it reflects the most common symptom of the disease. The international study group on CFS has decided to keep this name until a specific cause or marker of the disease is discovered, which may suggest a new name.

The CFS is called by different names in various parts of the world as:

i) Epidemic neuromyasthenia;

ii) Myalgic encephalomyelitis;

iii) Postviral fatigue syndrome;

iv) Chronic fatigue and immune system dysfunction syndrome.

In addition to the symptomatologic picture of the CFS that connects the two cases exposed there is the concomitant viral herpetic infection.

CMV and EBV are members of the herpes viridae family, which usually cause symptom-free infections. Characteristic of this family of viruses is that it no longer leaves the host after the first infection and nests in a cell type of the organism, causing a socalled latent infection. This occurs in a variable time depending on the type of virus and the sensitivity of the host. From this latency state the virus can reactivate, even after many years, resulting in a recurrence of the disease. The stimuli that induce the awakening of viral activity can be heat, cold, trauma, fever, stress and especially changes in the immune defense status of the host. All herpes viruses are responsible for productive and lytic infections, which after a more or less long phase depending on the reactivity of the infected person, turn into latent infections.

The latency site is different for each subfamily of herpes viruses, but they are always areas of the body protected from constant aggression of the immune system, which makes the eradication of these viruses from the infected subject virtually impossible.

In the clinical case of our study it was interesting to note, at a serological level, the negative values of the Extractable Nuclear Antigen Antibodies after treatment of oxygen-ozone therapy in synergy with the immunostimulating and protective cellular activity from oxidative stress of the Micetrin supplement used.

In the context of the controversial etiology of the CFS with a multifactorial infectious and non-infectious pathogenesis, oxygenozone therapy plays a fundamental role because of its direct and indirect action mechanism:

i) Direct action, in local application, of disinfectant and trophic type.

ii) Antibacterial and systemic antiviral effect due to peroxide formation.

iii) Increased deformability of red blood cells.

iv) Increased production of 2-3 diphosphoglycerides, at the level of the red blood cells, responsible for the $\mathrm{O}_{2}$ transfer to tissues.

v) Improvement of $\mathrm{O}_{2}$ metabolism in red blood cells by increasing glucose utilization, fatty acid cleavage and activation of enzymes that block peroxides and free radicals.

The systemic antiviral and antibacterial effects are responsible for a powerful bactericidal, fungicidal and viral inactivation action, which is achieved through the oxidation of microorganisms. The antiseptic mechanism is similar to the one the body usually uses with the formation, by the leukocytes used for bacterial phagocytosis, of a molecule with oxidizing properties, similar to that of $\mathrm{O}_{3}$.

Other important actions are the effects of ozone peroxides in phagocytosis. In fact, during infections, normal defensive processes are no longer able to destroy bacteria due to insufficient formation of $\mathrm{H}_{2} \mathrm{O}_{2}$. It is at this level that the positive influence of the peroxides formed during ozone treatment is highlighted.

The effects on the deformability of red blood cells, on the formation of 2.3 diphosphoglycerates (intermediate metabolite of erythrocytic glycolysis) and on the metabolism of $\mathrm{O}_{2}$ in erythrocytes are also important for the circulatory action of ozone and ultimately induce a marked improvement in the transport of $\mathrm{O}_{2}$. 
Peroxide formation facilitates direct activation of erythrocyte metabolism. The first step of the reaction consists in the interaction of ozone with the double bonds of unsaturated fatty acids of the phospholipid layer in the erythrocyte membrane. With the intervention of the glutathione system, an activation of the glycolysis takes place, which determines an increase of the 2,3-diphosphoglycerate, with relative facilitation of the release of $\mathrm{O}_{2}$ in the tissues by the hemoglobin:

$$
\mathrm{HbO}_{2}+2,3 \mathrm{DPG} \leftrightarrow \mathrm{Hb} * 2,3 \mathrm{DPG}+\mathrm{O}_{2}
$$

Therefore, also the effect of the ozone in the metabolism of erythrocytes could play an important role in the treatment of the CFS, being hypotension and anemia considered a clinical condition of the disease.

From these observations and from the clinical experience on patients affected by CFS treated daily in our clinics, we can affirm that the Oxygen-Ozone Therapy is the only effective therapeutic device in the neurological and functional aftermath of PostInfectious Meningoencephalitis Syndromes.

\section{References}

1. Hickie I, Davenport T, Wakefield D, Dubbo Infection Outcomes Study Group. Post-infective and chronic fatigue syndromes precipitated by viral and non-viral pathogens: prospective cohort study. BMJ 2006;333:575.

2. Fukuda K, Straus SE, Hickie I, et al. The chronic fatigue syndrome: a comprehensive approach to its definition and study. Ann Intern Med 1994;121:953-9.
3. Afari N, Buchwald D. Chronic fatigue syndrome: a review. Am J Psychiatry 2003;160:221-36.

4. Kaushik N, Fear D, Richards SCM, et al. Gene expression in peripheral blood mononuclear cells from patients with chronic fatigue syndrome. J Clin Pathol 2005;58:826-32.

5. Tirelli U, Cirrito C, Pavanello M. Ozone therapy is an effective therapy in chronic fatigue syndrome: result of an Italian study in 65 patients. Ozone Ther 2018;3:27-30.

6. Rilling S, Viebahn R. The use of Ozone in Medicine. In: Haug KF, ed. Heidelberg: VFM Publications; 1987. pp 7-187.

7. Buckey RD, Hackney JD, Clark K, Posin C. Ozone and human blood. Arch Envir Health 1975;30:40.

8. Begin ME. Fatty acids, lipid peroxidation and diseases. Proc Nutr Soc 1990;49:261-7.

9. Molinari F, Simonetti V, Franzini M, et al. L'autoemoterapia ozonizzata induce cambiamenti metabolici cerebrali a lungo termine nei pazienti affetti da sclerosi multipla. J Immunopathol Pharmacol 2014;379-89.

10. Oliviero F. Antioxidant and anti-inflammatory properties of a Shiitake-Maitake-Reishi-Cordyceps-based preparation for the prevention of oxidative stress and autoimmune diseases. The 9th International Medicinal Mushrooms Conference (IMMC9), September 24-28, 2017, Palermo, Italy. pp 109-110.

11. Scaglione G, Alio W, Maiorana A, Prestileo T. The M.O.P. therapy in HPV-related diseases of the female and male genital tract. A prospective observational clinical study. The 9th International Medicinal Mushrooms Conference (IMMC9), September 24-28, 2017, Palermo, Italy. pp 112-113.

12. Crosta L, Galanti D, Catarella MT, Valerio MR. Micetrin to prevent nausea and asthenia induced by chemotherapy in adjuvant treatment of breast cancer. The 9th International Medicinal Mushrooms Conference (IMMC9), September 2428, 2017, Palermo, Italy. pp 135-137. 